both APRIL and BAFF, it remained to be determined, whether this effect was due to the capacity of TACI to block just one or both ligands.

Methods CIA was induced in APRIL-transgenic (Tg) DBA/1 mice and littermates. Severity of disease was scored for each paw using a scale $0-4$. In addition, mice were analysed for histological signs of arthritis. Anticollagen antibody titers were determined by ELISA. Lymphocyte populations in draining lymph nodes, spleen and peritoneum were analysed by FACS. In another experimental setting mice were exposed to the collagen antibodies induced arthritis (CAIA). In addition, we employed models fort contact hypersensitivity (CHS) and experimental autoimmune encephalomyelitis (EAE). For the former, APRIL Tg and control mice were sensitised and challenged at the ear with oxazolone. EAE was induced by immunising mice with MOG, a myelin peptide, in adjuvant.

Results APRIL Tg mice displayed in contrast to littermates a lower disease score and incidence of arthritis, and also produced less collagen specific antibodies. Joints of littermates had higher IgG levels and increased number of degranulating, thus activated, mast cells. To confirm that the decreased IgG levels developed in the CIA model in APRIL Tg mice are directly linked to the less severe disease development, we employed the model of CAIA. In fact, disease development in CAIA was similar in APRIL Tg and control mice. In addition, we detected a significantly increased IL-10 production of peritoneal B cells in APRIL Tg mice in the CIA model. Evidence is accumulating that IL-10 producing B cells can regulate autoimmune diseases including arthritis. The regulatory role of APRIL by modulating activity IL-10-producing B was confirmed in the CHS and EAE model.

Conclusion Our results show that APRIL can control inflammation in the three tested disease models CIA, CHS and EAE. This suggests a therapeutic potential of APRIL agonists to downregulate inflammatory diseases such as rheumatoid arthritis.

\section{A135 THE TNF-LIGAND APRIL CAN CONTROL CIA BY REGULATING ANTIBODY-PRODUCTION AND STIMULATING ANTI-INFLAMMATORY IL-10 PRODUCING B CELLS}

Leticia Fernandez, ${ }^{1-3}$ Gabriela Franco Salinas, ${ }^{4}$ Cecilia Rocha, ${ }^{1-3}$ Carla Carvalho, ${ }^{5}$ Nataliya Yeremenko, ${ }^{4}$ Jan Paul Medema, ${ }^{4}$ Bernard Combe, ${ }^{1,2}, 6$ Dominique Baeten, ${ }^{4}$

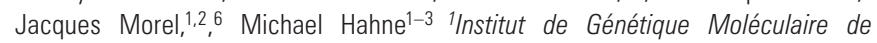
Montpellier, CNRS, UMR5535, Montpellier, France; ${ }^{2}$ Université Montpellier 1, Montepellier, France; ${ }^{3}$ Université Montpellier 2, Montepellier, France; ${ }^{4}$ Academic Medical Center, University of Amsterdam, Amsterdam, The Netherlands, ${ }^{5}$ Department

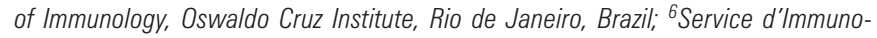
Rheumatologie, CHU Lapeyronnie, Montepellier, France

\subsection{6/ard.2010.149005.2}

Background Increased levels of the tumour necrosis factor (TNF) -ligand APRIL (A Proliferation Inducing Ligand) were found in synovial fluid and serum of patients with inflammatory arthritis pointing to a pro-inflammatory role of APRIL. APRIL can bind to BCMA and TACI, two receptors of the TNF family, which can also bind the $B$ cell activating factor (BAFF). In the collagen-induced arthritis (CIA), administration of TACI-Ig was found to prevent disease progression and to lower disease scores, compared with controls. As TACI binds 